\title{
A Dynamic Adjustment Method of Service Function Chain Resource Configuration
}

\author{
Xiaoyang Han ${ }^{1}$, Xiangru Meng1, Zhenhua $\mathbf{Y u}^{2 *}$ and Dong Zhai ${ }^{1}$ \\ ${ }^{1}$ College of Information and Navigation, Air Force Engineering University \\ Xi'an, Shaanxi 710077 China \\ [e-mail:dodogg189888@163.com] \\ ${ }^{2}$ College of Computer Science and Technology, Xi'an University of Science and Technology \\ Xi'an, Shaanxi 710054 China \\ [e-mail: zhenhuayu@xust.edu.cn] \\ ${ }^{*}$ Corresponding author: Zhenhua $\mathrm{Yu}$
}

Received January 4, 2021; revised May 27, 2021; revised June 30, 2021; accepted August 1, 2021; published August 31, 2021

\begin{abstract}
In the network function virtualization environment, dynamic changes in network traffic will lead to the dynamic changes of service function chain resource demand, which entails timely dynamic adjustment of service function chain resource configuration. At present, most researches solve this problem through virtual network function migration and link rerouting, and there exist some problems such as long service interruption time, excessive network operation cost and high penalty. This paper proposes a dynamic adjustment method of service function chain resource configuration for the dynamic changes of network traffic. First, a dynamic adjustment request of service function chain is generated according to the prediction of network traffic. Second, a dynamic adjustment strategy of service function chain resource configuration is determined according to substrate network resources. Finally, the resource configuration of a service function chain is pre-adjusted according to the dynamic adjustment strategy. Virtual network functions combination and virtual machine reusing are fully considered in this process. The experimental results show that this method can reduce the influence of service function chain resource configuration dynamic adjustment on quality of service, reduce network operation cost and improve the revenue of service providers.
\end{abstract}

Keywords: Service function chain, Virtual network function, Dynamic adjustment, Vertical scaling, Migration 


\section{Introduction}

$\mathbf{N}_{\text {etwork function virtualization (NFV), which is based on virtualization technology and }}$ standard commodity hardware instead of a dedicated middle box to realize network functions, decouples network functions completely from the dedicated hardware devices, supports fast launch of emerging services, greatly improves the flexibility of service deployment and reduces network operation cost $[1,2]$. The service requests of network function virtualization are usually deployed in the form of a service function chain (SFC). Virtual network functions (VNFs) deployed on network infrastructure in a predefined order are combined into a virtual path to provide users with corresponding network services [3, 4]. At present, many researchers have made unremitting efforts on SFC deployment and achieved many research results. The work in [5] proposes a new SFC resource configuration method aiming at energy efficiency and quality of service (QoS) assurance, which improves the SFC deployment performance and has a good practical application. The work in [6] studies the SFC deployment in the internet of things on the basis of ensuring QoS, and proposes a resource and QoS awareness method of SFC deployment.

Dynamic adjustment methods for SFC resource configuration include vertical scaling, horizontal scaling and VNF migration [7]. The work in [8] deals with the dynamic changes of network traffic by revoking or reinstantiating VNFs, to ensure network load balancing and prevent network congestion, which is also known as horizontal scaling. However, this coarsegrained adjustment method will lead to resource overconfiguration and inevitable service interruption. Instead of adding or removing VNF instances, the vertical scaling responds to dynamic changes in network traffic by increasing or decreasing the capacity of VNF instances while keeping the VM running continuously. Compared with the horizontal scaling, the vertical scaling has a shorter service interruption time. An elastic VNF resource configuration and adjustment method is proposed in [9], which uses a hybrid method of vertical scaling and horizontal scaling to dynamically adjust SFC, thereby reducing service interruption time and avoiding resource overconfiguration.

Due to the limited capacity of server nodes, VNF migration is an effective method to deal with the overloading of server nodes. Many researchers have conducted researches on VNF migration. The study in [10] designs a load-aware NFV state management method for traffic load optimization. The hidden Markov method is used to predict link load and make adjustment actively. However, this method does not consider the changes of VNF demand caused by traffic fluctuations. The work in [11] jointly optimizes SFC deployment and dynamic resource configuration, but does not consider the service interruption time and network operation cost of SFC resource adjustment.

This paper studies the SFC dynamic adjustment based on VNFs deployment in VMs. There are several problems such as long service interruption time and high network operation cost in current researches. VNFs combination and VM reusing technology are not fully considered in the process of SFC dynamic adjustment, and there is still room for improvement. A dynamic adjustment method of SFC resource configuration (DA-SFC) is proposed in this paper based on the assumption that the network traffic is dynamic. First, a traffic prediction algorithm in the work of [12] is used to predict the traffic dynamic changes in advance, and duration thresholds are set to generate SFC dynamic adjustment requests. Second, according to an SFC dynamic adjustment request, a proactive SFC dynamic adjustment strategy is generated and VNFs combination and VM reusing are preferred in VNF migration. The experimental results show that the proactive fine-grained resource configuration adjustment strategy can reduce 
service interruption time and adjustment operation cost, and improve the revenue of service providers.

The main contributions of this paper can be summarized as follows.

- A proactive adjustment method. According to network traffic prediction, the proactive adjustment of SFC resource configuration can reduce service interruption time and impact on QoS, thus reducing the penalty of service providers.

- A fine-grained adjustment method. SFC adjustment requests are generated by setting the thresholds of fluctuation duration, so as to avoid the frequent adjustment of SFC resource configuration, thus reducing network operation cost. In addition, priority is given to vertical scaling, and VNF migration is considered in SFC resource configuration adjustment when service demands cannot be met. These measures can greatly reduce adjustment operation cost.

- VNFs combination and VM reusing technology are fully considered in the process of SFC dynamic adjustment to improve resource utilization, thus improving the adjustment success rate of SFC.

The rest of this paper is organized as follows. In Section 2, some related works are reviewed. Section 3 gives the problem statement. In Section 4, the integer linear programming formulation of SFC dynamic adjustment is presented. DA-SFC is elaborated in Section 5. Section 6 validates and evaluates the proposed method with extensive simulations and experiments. We conclude this paper in Section 7.

\section{Related Work}

The dynamic changes of network traffic need to dynamically adjust SFC resource configuration, and designing a dynamic SFC resource configuration adjustment method has become a realistic requirement of NFV application. This section reviews some SFC resource configuration dynamic adjustment researches.

\subsection{VNF vertical scaling and VNF migration}

VNF vertical scaling provides VNF instances with the ability to adjust load online, ensures shorter adjustment time (milliseconds) and faster service response, thus guaranteeing QoS and resource utilization. As a vertical scaling technology, the kernel-based VM technology (KVM) known as a hot plug technology can be used to dynamically adjust CPU resources in kernelbased VMs [13]. The KVM control platform can dynamically adjust the CPU resources of VMs and the normal operation of VMs is not affected. Research shows that the KVM technology needs 40ms to scale up the CPU resource of VNFs, while it only needs 20ms to scale down the CPU resource of VNFs [14]. Additionally, open vswitch technology can be used to realize the rapid scaling of virtual link resources in milliseconds [15].

VNF migration arises from VM migration, but there are some differences between them. Traditional VM migration only needs to consider the offline state of VMs, so VMs can be shut down directly before migration, while VNF migration needs to consider the online state of VNFs and the end-to-end performance demands of SFCs [16]. The work in [17] studies the VM startup time of three cloud service providers, Amazon EC2, Microsoft Azure and Rackspace. The VM startup times of EC2, Azure and Rackspace are 96.6s, 356.6s and 44.2s, respectively. Research in [18] shows that the link bandwidth deployment time between VMs is microseconds, and compared with the VM startup time, the link bandwidth deployment time is negligible. VNF migration is shown in Fig. 1. When $\mathrm{VNF}_{2}$ fails or overloads, it can be 
migrated to other server nodes and the corresponding virtual links can be deployed. It is necessary to ensure that its state is transferred simultaneously to reduce the impact on QoS.

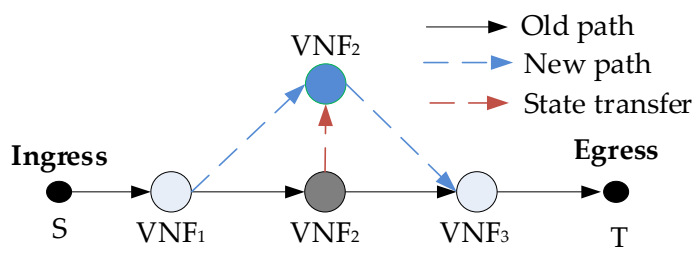

Fig. 1. Example of VNF migration

VNF migration can realize load balancing, rapid recovery of failures and rapid response to dynamic changes of network services [19]. In [20], a node-aware and link-aware VNF migration method is designed to migrate the VNFs which are deployed on overloaded server nodes. This method does not consider VNFs combination in the process of VNF migration, and the performance still has room for improvement. The work in [21] establishes an integer nonlinear programming model for the joint optimization of SFC deployment and adjustment. The solution results show that this method could reduce the adjustment operation cost on the basis of guaranteeing QoS.

\subsection{VNFs combination and VM reusing}

Mehraghdam et al. [22] propose methods of VNFs combination and VM reusing, which provide new ideas for improving resource utilization and shortening the link length of SFC deployment. In fact, the load of VNFs is not always full. Therefore, multiple same type VNFs of different SFCs could share the same VM resources through time division multiplexing for each SFC packet processing in the NFV environment, thus significantly improving the resource utilization of server nodes. As shown in Fig. 2, flow1 includes $\mathrm{VNF}_{3}$ and $\mathrm{VNF}_{2}$, and the deployment path is (B - E(e) - C(c) - D); flow2 includes $\mathrm{VNF}_{1}$ and $\mathrm{VNF}_{2}$, and the deployment path is $(\mathrm{B}-\mathrm{A}(\mathrm{a})-\mathrm{C}(\mathrm{c})-\mathrm{D})$. $\mathrm{VNF}_{2}$ is deployed on the $\mathrm{VM}$ of server node $\mathrm{c}$, so $\mathrm{VM}$ reusing can be considered to improve resource utilization. Set the polling period of a VM as $T_{0}$, the time share for flow1 as $t_{1}$, and the time share for flow2 as $t_{2}$, the context switching time as $t_{s}$, and the residual available time of a VM as $t_{r}$.

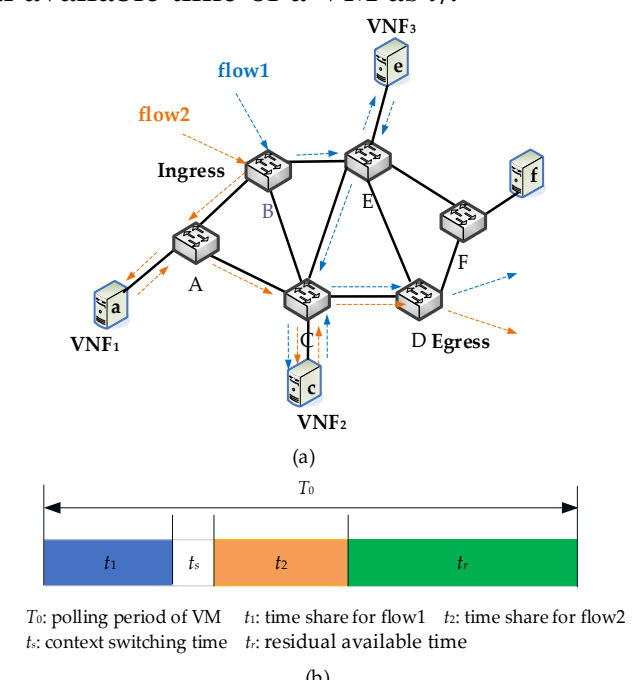

(b)

Fig. 2. Description of VM resuing method:(a) Example of VM reusing;(b) Description of VM reusing 
VNFs combination means that multiple adjacent VNFs of an SFC can be deployed on the same server node under the condition of meeting resource constraints and VNF types constraints. VNFs combination and VNF resuing could reduce network operation cost and improve resource utilization in VNFs migration.

\subsection{SFC resource configuration adjustment}

At present, most studies on SFC resource configuration adjustment are movitated by the changs of SFC resource demands. The service interruption time is long, and it is hard to meet real needs [23]. Predicting the dynamic changes of network traffic, determining the dynamic adjustment demand of SFC resource configuration, and carrying out the SFC dynamic adjustment in advance, can effectively deal with the degradation of QoS. The work in [24] proposes a delay-aware flow migration strategy for a delay sensitive flow migration problem, which can predict the resource demand of SFC in advance according to a network traffic prediction and make adjustments by using VNF and link migration methods, so as to reduce the service interruption time and adjustment operation cost as far as possible. The work in [25] proposes a VNF migration method based on traffic prediction, which uses a deep learning method to predict the dynamic changes of network traffic, and carries out proactive VNF and link migration according to the traffic prediction to ensure QoS. The work in [26] studies VNF dynamic scaling in a data center network, and a sliding window method is used to predict the peak value of traffic demand at the next moment, and then VNF dynamic adjustment is carried out according to the prediction to ensure high availability. Luo et al. [27] aim at the deployment and dynamic adjustment of geographically distributed SFCs, and a circular neural network is used to predict the dynamic changes of network demand in advance, and make SFC dynamic adjustment according to the prediction. The coarse-grained dynamic resource configuration adjustment methods proposed above have some problems, such as high adjustment operation cost and low resource utilization.

Aiming at the problem of high adjustment operation cost caused by coarse-grained dynamic adjustment methods, some researchers attempt to make fine-grained dynamic adjustment for the SFC resource configuration [9, 28, 29]. A fine-grained adjustment method for the SFC resource configuration is proposed in [28] and achieves good results. Yu et al. [29] propose a fine-grained resource configuration adjustment method, which gives priority to solving the dynamic change of traffic through VNF vertical scaling. VNF migration is only carried out when VNF vertical scaling cannot meet the demands. Fine-grained adjustment can avoid resource over-configuration and reduce service interruption time. However, these studies fail to consider the VM reusing and VNFs combination in VNF migration, and there is still room for improvement in the success rate and resource utilization.

According to the above analysis, VNF vertical scaling can significantly shorten adjustment time and reduce adjustment operation cost compared with VNF migration. The proactive SFC dynamic adjustment strategy, which adopts a hybrid approach of VNF vertical scaling and migration in response to the dynamic changes of network traffic, and fully consideres VNFs combination and VM reusing in the adjustment process, can better meet the real needs of dynamic resource reconfiguration. This is also the motivation of this paper. 


\section{Problem Statement}

\subsection{SFC dynamic adjustment}

In order to deal with the problems of low revenue and high adjustment operation cost in SFC dynamic adjustment, this paper proposes an SFC dynamic adjustment method for the dynamic changes of network traffic. First, the research results of our team are used to accurately predict the changes of network traffic. According to the traffic prediction, thresholds are set to generate SFC dynamic adjustment request, so as to prevent the ping-pong effect caused by frequent changes of resource configuration. Second, according to the network resource constraints and SFC dynamic adjustment request, the SFC dynamic adjustment strategy can be determined: VNF vertical scaling or VNF migration. Finally, in the VNF migration process, as VNFs combination and VM reusing can effectively improve resource utilization rate and reduce link transmission delay, these two technologies are considered in the VNF migration process to improve adjustment success rate and resource utilization.

\subsection{Evaluation indicators}

The performance of the proposed method is evaluated by four evaluation indicators: adjustment success rate, average link expansion coefficient, average adjustment operation cost and average penalty.

\subsubsection{Adjustment success rate}

The adjustment success rate is an important indicator to evaluate an SFC dynamic adjustment method. In this paper, the adjustment success rate is defined as the ratio of the SFC adjustment requests that are successfully adjusted within a predicted time to all the SFC adjustment requests that arrive, which can be defined as follows:

$$
r=\lim _{T \rightarrow \infty} \frac{\sum_{t=0}^{T} S C_{\text {suc }}(t)}{\sum_{t=0}^{T} S C(t)+\delta}
$$

where $S C_{\text {suc }}(t)$ represents the number of SFC adjustment requests successfully adjusted at time $t, S C(t)$ represents the number of SFC adjustment requests arriving at time $t$, and $\delta$ is a constant close to 0 .

\subsubsection{Average link expansion coefficient}

The average link expansion coefficient is also an important indicator to evaluate the performance of an SFC dynamic adjustment method. In this paper, the average link expansion coefficient is defined as the ratio of the sum of the substrate path hops of virtual links deployment to the sum of the virtual link hops in all successful SFC adjustment requests, which can be defined as follows:

$$
K=\frac{\sum_{e_{v}^{i j} \in E_{v}} \operatorname{hops}\left(D\left(e_{v}^{i j}\right)\right)}{\sum_{e_{v}^{i j} \in E_{v}} \operatorname{hops}\left(e_{v}^{i j}\right)}
$$


where $D\left(e_{v}^{i j}\right)$ represents the substrate path of virtual link $e_{v}{ }^{i j}$ deployment, $E_{v}$ represents the virtual links set of an SFC, $\sum_{e_{v}^{i} \in E_{v}} h o p s\left(D\left(e_{v}^{i j}\right)\right)$ represents the sum of the substrate path hops of virtual link $e_{v}^{i j}$ deployment, and $\sum_{e_{v}^{i j} \in E_{v}} h o p s\left(e_{v}^{i j}\right)$ is the sum of the virtual link hops of successful SFC adjustment requests.

\subsubsection{Average adjustment operation cost}

In the process of SFC dynamic adjustment, VNF vertical scaling and virtual link vertical scaling only need software defined network controllers to adjust and reallocate network resources online. As a result, the operation costs of a VNF and a link vertical scaling are low, and can be denoted as $S c_{1}$ and $S c_{2}$, respectively. VNF migration needs to send all packets received by a VNF to a software defined network controller until the end of VNF migration. Therefore, the adjustment operation cost of VNF migration refers to the adjustment operation cost of forwarding traffic to a controller. For the sake of brevity, this paper takes the sum of the migrated VNFs and rerouted links as the adjustment operation cost, where $M c_{1}$ represents a VNF migration cost and $M c_{2}$ represents a link rerouting cost.

For the hybrid approach of VNF vertical scaling and migration proposed in this paper, the total operation cost of the $i$-th SFC adjustment request can be defined as follows:

$$
M S_{i}=\left(x_{1} \times S c_{1}+x_{2} \times S c_{2}+y_{1} \times M c_{1}+y_{2} \times M c_{2}\right)
$$

where $x_{1}, x_{2}$ represent the number of VNFs vertical scaling and links vertical scaling in the $i$ th SFC adjustment request, respectively, and $y_{1}, y_{2}$ represent the number of migrated VNFs and rerouted links in the $i$-th SFC adjustment request, respectively.

The average adjustment operation cost can be defined as follows:

$$
\overline{M S}=\frac{\sum_{i=1}^{N_{1}} M S_{i}}{N_{1}}
$$

where $N_{1}$ is the total number of adjustment requests within the entire experimental period $T$, and $M S_{i}$ is the adjustment operation cost of the $i$-th SFC adjustment request.

\subsubsection{Average penalty}

This paper defines average penalty as the penalty paid by service providers per unit time. Average penalty can reflect the overall revenue of service providers. The penalty paid by the service providers shall include penalty for failure to generate adjustment requests due to traffic fluctuations, penalty for failure to dynamically adjust an SFC, and penalty for VNF and link migration. We define the penalty paid by service providers per unit time for QoS degradation caused by failure to generate adjustment requests due to traffic fluctuations as $\beta_{1}$, and the penalty paid by service providers per unit time for QoS degradation caused by failure to dynamically adjust an SFC as $\beta_{2}$. Since VNF and link migration result in reduced QoS, the penalty paid by service providers per unit time due to VNF and link migration is $\beta_{3}$. The average penalty can be defined as follows: 


$$
\overline{\text { Penalty }}=\frac{\beta_{1} \times \sum_{i=1}^{N_{0}} t_{i}+\beta_{2} \times \sum_{j=1}^{N_{1}} t_{j}+\beta_{3} \times N_{2}}{T}
$$

where, $t_{i}$ is the duration of the $i$-th SFC fluctuation, $t_{j}$ is the duration of the $j$-th SFC request, $N_{0}$ is the number of the SFC dynamic flow fluctuation which does not generate adjustment requests within the entire experimental period $T$, and $N_{2}$ is the number of VNF and link migration within the entire experimental period $T$.

\section{Mixed integer linear programming model}

\subsection{Objective function}

The purpose of this paper is to improve the revenue of service providers on the basis of guaranteeing QoS. The average link expansion coefficient and adjustment success rate can reflect the impact on the QoS. The average penalty can reflect the revenue of service providers. The average adjusting operation cost can not only reflect the impact on the QoS, but also indirectly reflect the revenue of service providers. The SFC adjustment method proposed in this paper makes proactive adjustment in advance and gives priority to the use of vertical scaling, which can reduce the average adjustment operation cost, and thus reducing the impact on QoS and improving the revenue of service providers. Therefore, reducing the average adjustment operation cost is selected as the optimization goal, which can be expressed as follows:

$$
\min \overline{M S}
$$

\subsection{Constraints}

$$
\begin{aligned}
& x_{i}^{l}=\left\{\begin{array}{ll}
1, & \text { if } v_{i} \text { need to be adjusted } \\
0, & \text { otherwise }
\end{array},\right. \\
& \forall v_{i} \in V \\
& y_{i j}^{l m}= \begin{cases}1, & \text { if } e_{v}^{i j} \text { needs to be adjusted } \\
0 & \text { otherwise }\end{cases} \\
& \forall e_{v}^{i j} \in E_{v}
\end{aligned}
$$

Constraint (7) indicates that whether the VNF $v_{i}$ deployed on the server node $n_{c}{ }_{c}^{l}$ needs to be adjusted for resource configuration, $x_{i}^{l}=1$. Otherwise, $x_{i}^{l}=0$, and $V$ represents the VNFs set of an SFC. Constraint (8) means that if the virtual link $e_{v}^{i j}$ deployed on the substrate network link $e_{s}^{l m}$ needs to be adjusted for resource configuration, $y_{i j}^{l m}=1$. Otherwise, $y_{i j}^{l m}=0$.

$$
\begin{gathered}
\sum_{v_{i} \in V} x_{i}^{l} \leq m, \quad \forall n_{c}^{l} \in N_{c} \\
\sum_{n_{c}^{l} \in N_{c}} x_{i}^{l}=1, \quad \forall v_{i} \in V
\end{gathered}
$$




$$
\begin{gathered}
x_{i}^{l} \times c p u\left(v_{i}\right) \leq \operatorname{cpu}\left(n_{c}^{l}\right), \\
\forall v_{i} \in V, \forall n_{c}^{l} \in N_{c} \\
\sum_{e_{v}{ }^{i j} \in E_{v}} y_{i j}{ }^{l m} \times b w\left(e_{v}^{i j}\right) \leq b\left(e_{s}^{l m}\right), \\
\forall e_{v}^{i j} \in E_{v}, \forall e_{s}^{l m} \in E_{s} \\
\sum_{e_{v}^{i j} \in E_{v}} y_{i j}{ }^{l m} \times b w\left(e_{v}^{i j}\right) \leq \text { forward }\left(n_{f}{ }^{l}\right), \\
\forall n_{f}{ }^{l} \in N_{f}, \forall e_{v}^{i j} \in E_{v}, \forall e_{s}^{l m} \in E_{s} \\
z_{i}^{l}=\left\{\begin{array}{l}
1, \text { if } f_{i} \text { is satisfied by } n_{c}{ }^{l}, \\
0, \text { otherwise }
\end{array}\right. \\
\forall v_{i} \in V, \forall n_{c}^{l} \in N_{c}
\end{gathered}
$$

Constraint (9) denotes that the number of VNF types that each substrate node can host is $m$. The constraint (10) means that each VNF of an SFC can only be deployed on a server. Constraint (11) means that the residual available CPU resources $c p u\left(n_{c}{ }^{l}\right)$ of the server node $n_{c}{ }^{l}$ cannot be less than the CPU resource demand $c p u\left(v_{i}\right)$ of $v_{i}$, and $N_{c}$ represents the set of server nodes. Constraint (12) indicates that the residual bandwidth resource of the substrate link $e_{s}^{l m}$ should be greater than the link resource demand of SFC, and $E_{s}$ represents the set of substrate links. Constraint (13) means that the residual forwarding capacity of a switch node should be greater than the forwarding resource demand $b w\left(e_{v}^{i j}\right)$. forward $\left(n_{f}^{l}\right)$ means the residual available forwarding resources of the substrate switch node $n_{f}^{l}$, and $N_{f}$ means the set of forwarding nodes. The constraint (14) indicates that a VNF type demand must be satisfied by a server node, and $f_{i}$ represents the resource type demand of $v_{i}$.

$$
\begin{gathered}
\sum_{\substack{e_{s} m \in E_{s} \\
\forall}} y_{i j}^{l m}-\sum_{e_{s}^{m l} \in E_{s}} y_{j i}{ }^{m l}=x_{i}^{l}-x_{j}{ }^{m}, \\
D_{p} \leq d, \quad \forall p \in \sum_{t=0}^{T} S F C_{\text {suc }}(t) \\
Z_{1}^{l m}= \begin{cases}1, & \text { if } n_{c}{ }^{m} \text { is a neighboring node of } n_{c}{ }^{l}, \\
0, & \text { otherwise }\end{cases} \\
\forall n_{c}^{l} \in N_{c}, \forall n_{c}{ }^{m} \in N_{c} \\
Z_{2}{ }^{m}= \begin{cases}1, & \text { if } v_{m} \text { with reusable resources } \\
0 & \text { otherwise }\end{cases} \\
\forall v_{m} \in V \quad V
\end{gathered}
$$

Constraint (15) means that the virtual link should be deployed to the loopfree path of the substrate network to prevent the Ping-pong routing problem. Constraint (16) denotes that SFCs deployed successfully must meet the delay demands of service flows, $D_{p}$ represents the delay 
of the $p$-th SFC adjustment request that is successfully processed, and $d$ represents the delay constraint of an SFC. Constraint (17) indicates that if the server node $n_{c}{ }^{m}$ is the adjacent deployment node of $n_{c}{ }^{l}$ in an SFC, VNFs combination is selected in the migration process. Constraint (18) indicates that if $v_{m}$ has reusable resources, then VM reusing is preferred during the migration process.

\section{Dynamic adjustment method of SFC resource configuration}

\subsection{Process of a dynamic adjustment method of SFC resource configuration}

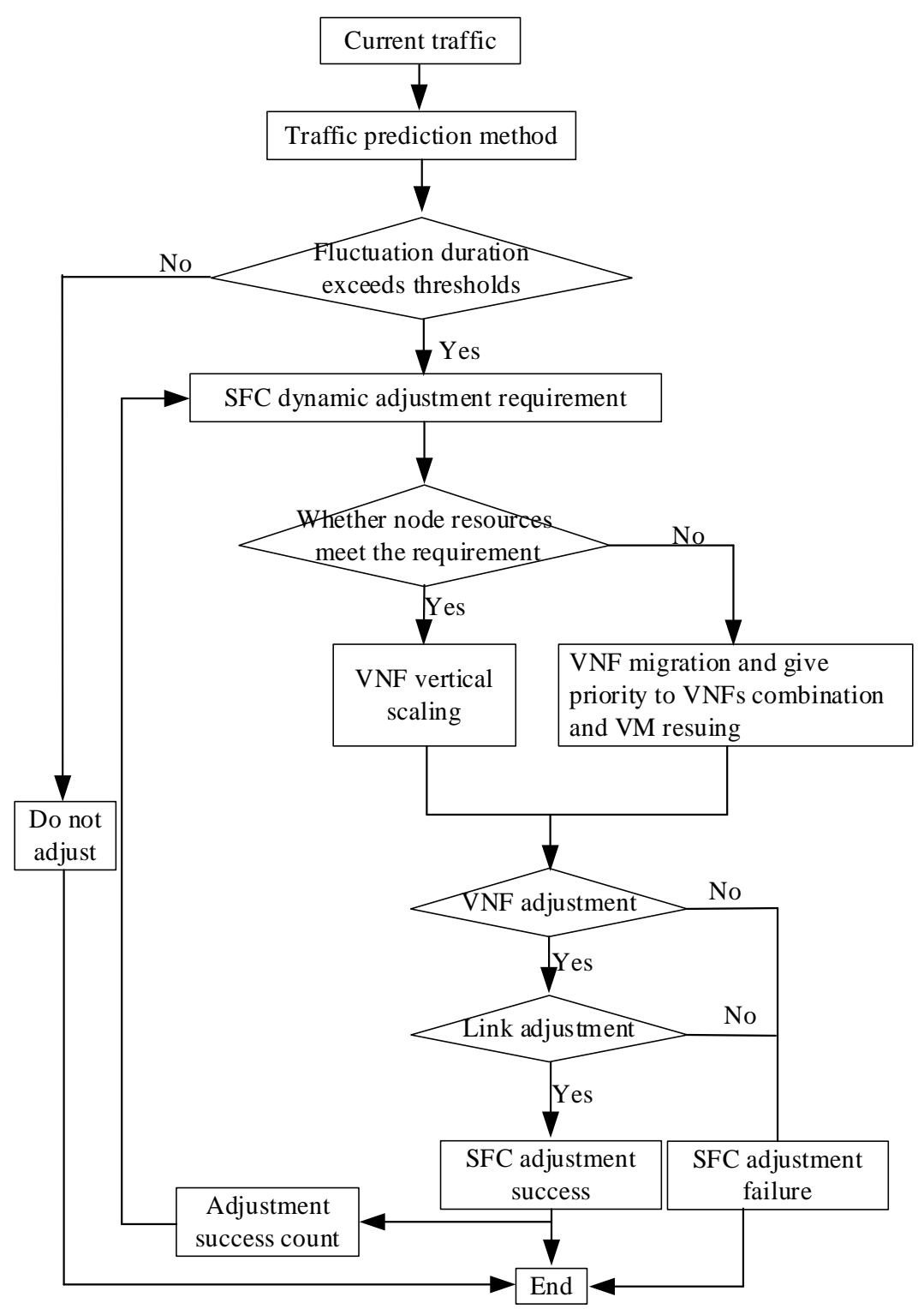

Fig. 3. Process of the dynamic adjustment method 
As shown in Fig. 3, a dynamic adjustment method of SFC resource configuration includes an SFC dynamic adjustment request generation method and an SFC dynamic adjustment mechanism. First, the traffic prediction method is used to accurately predict the changes of network traffic, and duration thresholds are set to generate SFC dynamic adjustment requests. Second, an SFC dynamic adjustment mechanism is designed to process SFC dynamic adjustment requests. Priority is given to vertical scaling. If vertical scaling cannot meet the demands, VNF migration and link rerouting will be used for adjustment. Finally, VNFs combination and VM reusing are fully considered in the migration process to improve resource utilization rate and adjustment success rate as much as possible.

\subsection{SFC dynamic adjustment request generation method}

The dynamic changes of network traffic reflect the SFC dynamic adjustment requirements, including VNF resource adjustment requirements and link resource adjustment requirements. If the dynamic changes of network traffic can be predicted in advance, proactive strategies can be taken to adjust SFC to effectively solve the problems such as service interruption caused by network traffic fluctuations. In this paper, the traffic prediction algorithm (HTPA-OPS) in the work of [12] is used to predict a network traffic. The prediction can fit well with the actual network traffic, and it is useful for the SFC dynamic adjustment. When the duration of flow fluctuation exceeds the thresholds which are set proactively, the SFC dynamic adjustment requests are generated. Otherwise, service degradation and payment of the corresponding penalty according to the service level agreement are considered. Setting the duration thresholds can prevent the ping-pong effect of frequent adjustment of resource configuration, and proactive adjustment of SFC resource configuration can reduce the impact on QoS as far as possible.

The network traffic prediction algorithm used in this paper has a high prediction accuracy within 150s, and the prediction fits well with real network traffic. Therefore, the prediction time is set as $T=150$ s and the service hosted by an SFC is selected as delay sensitive service (such as VOIP service, with delay constraint within 200ms). The duration threshold $C^{-}$of SFC adjustment request generation is set as $C^{-}=200 \mathrm{~ms}$ for the increased resource demand. In order to reduce the adjustment operation cost caused by frequent resource adjustment, the duration threshold $C$. is set as $C=1 \mathrm{~s}$ for the reduced resource demand. For the generated SFC adjustment requests, a fine-grained adjustment method is proposed to adjust resource configuration dynamically in this paper.

The specific steps of the SFC dynamic adjustment request generation method are as follows:

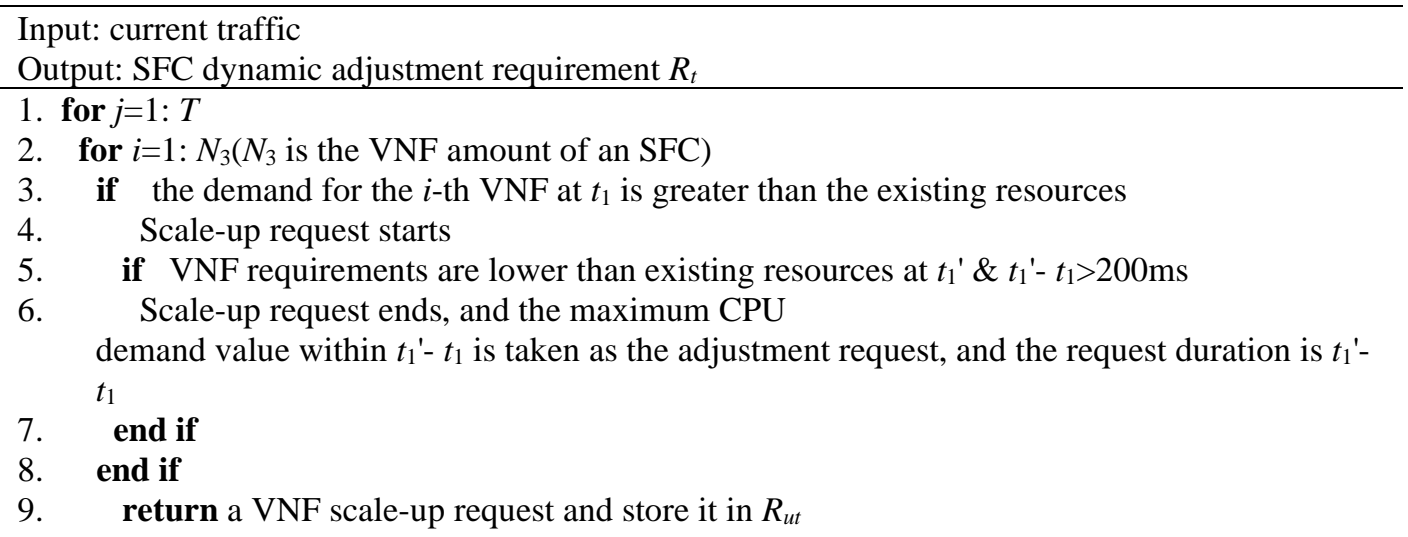




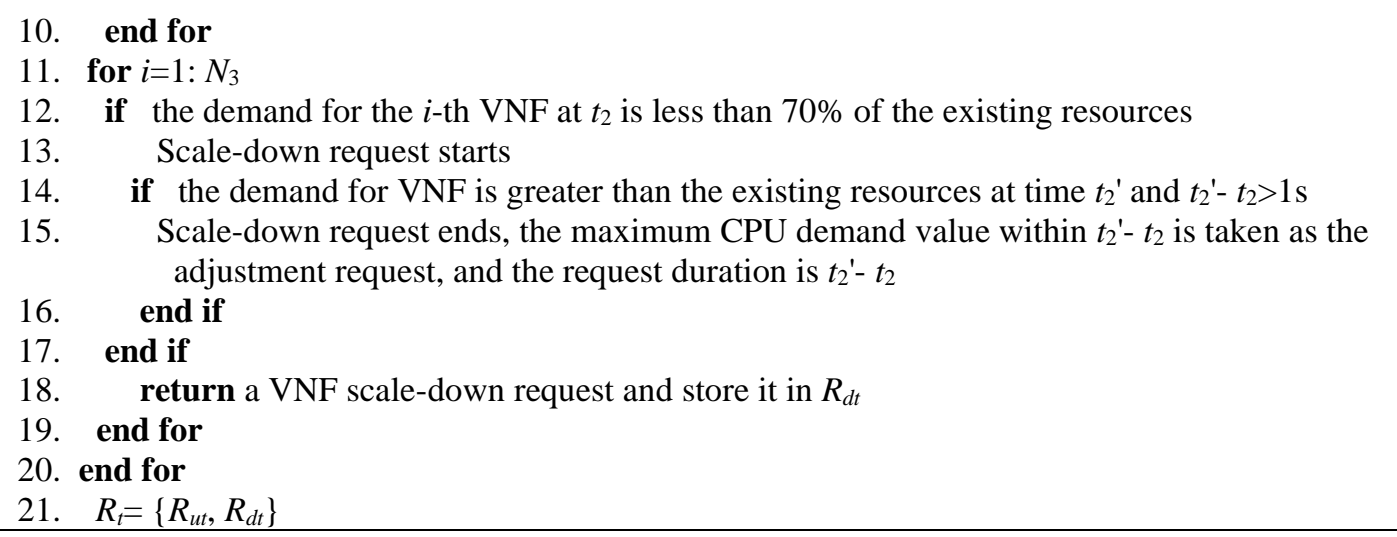

HTPA-OPS is used to predict dynamic changes of a network traffic, and $N_{3}$ represents the VNF amount of an SFC. Step (2-10) means when the demand for the $i$-th VNF at $t_{1}$ is greater than the existing resources and the duration is more than $200 \mathrm{~ms}$, a VNF scale-up request is generated. Step (11-19) means when the demand for the $i$-th VNF at $t_{2}$ is less than $70 \%$ of the existing resources and the duration is more than 1s, a VNF scale-down request is generated.

\subsection{SFC dynamic adjustment mechanism}

The SFC dynamic adjustment mechanism is triggered when an SFC dynamic adjustment request arrives, and an SFC adjustment strategy is determined according to the substrate network carrying capacity. If the node carrying capacity can meet the demand, VNF vertical scaling should be adopted for adjustment to reduce adjustment operation cost and service interruption time. VNF migration is selected when the substrate network has insufficient resources available for VNF adjustment through vertical scaling. When looking for a suitable migration node, priority is given to adjacent VNFs combination to reduce link bandwidth consumption. VM reusing is considered to improve resource utilization and reduce adjustment operation cost. After the dynamic adjustment of VNFs in an SFC is completed, the link vertical scaling method or rerouting mechanism is determined according to the link resources and the delay requirement to quickly adjust the link resource configuration to complete the SFC dynamic adjustment.

In the VNF migration process, VNF $v_{r}$ is selected to be migrated and its deployment node location $L o c_{r}$ is determined. First, the distance (link hops) between $v_{r-1}$ and deployment node $S^{\prime}$ (or the ingress switch node) is calculated as $L_{1}$, and the distance (link hops) between $L o C_{r}$ and $v_{r+1}$ deployment node $T$ (or the egress switch node) is calculated as $L_{2}$, and let $L=\operatorname{Max}\left(L_{1}\right.$, $L_{2}$ ). Second, with the $L o c_{r}$ as the center of a circle, $L$ as the radius, and VNF resources, types and deployment location requirements as constraints, each server node is traversed in the circle, and all feasible server nodes are stored into the candidate nodes set. Then, according to the candidate nodes set, a new SFC deployment path is generated under the condition that the delay and VNF position constraints are satisfied. Finally, the adjacent nodes in the SFC are preferred for VNFs combination to shorten path length, and VM reusing is also considered in this process. 
The specific steps of the SFC dynamic adjustment mechanism are as follows: Input: $R_{t}$

Output: optimal redeployment path $\boldsymbol{O R P}$

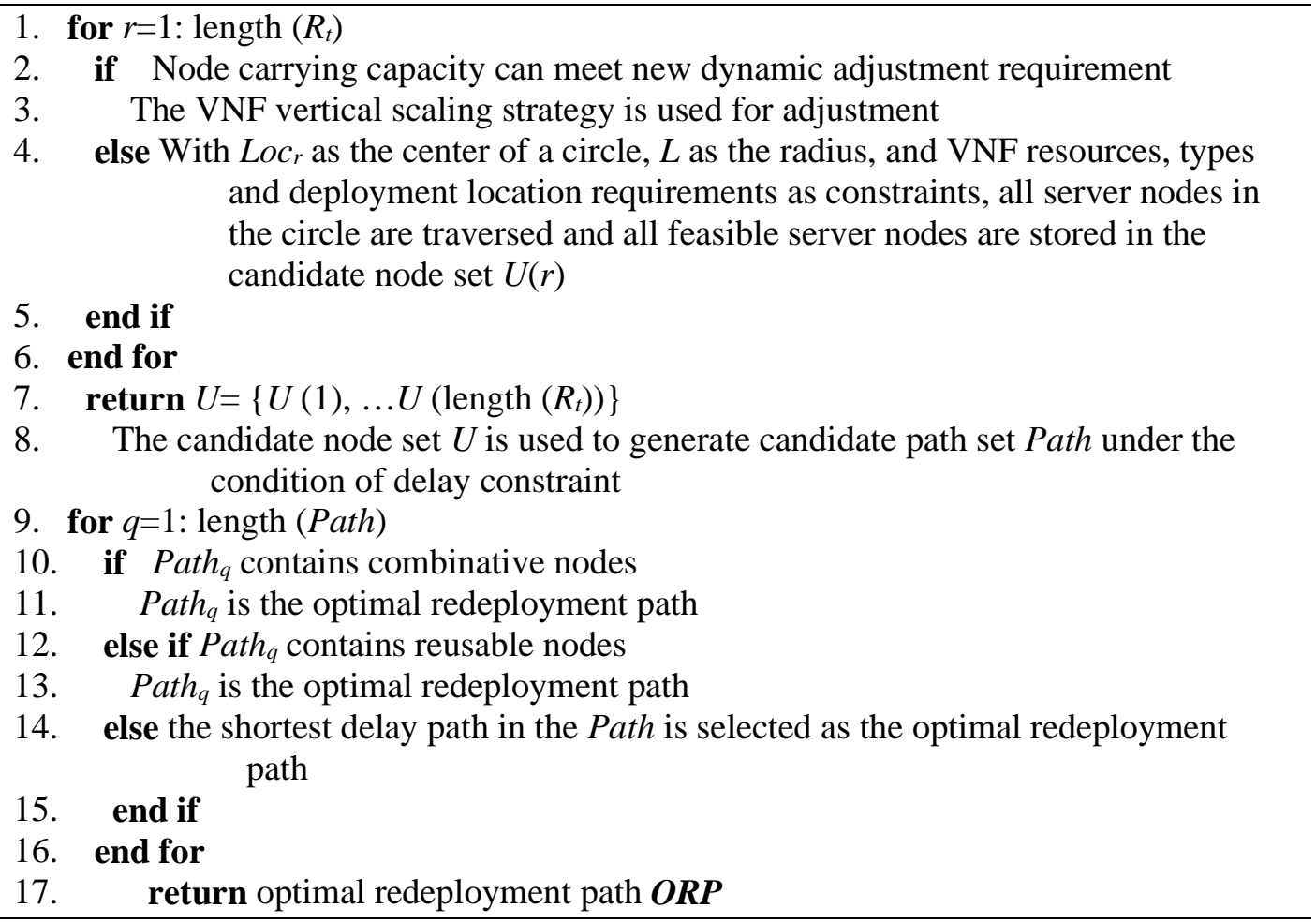

Step (1-6) is to determine the deployment location $L c_{r}$ of a VNF which needs to adjust. Step (7-8) means that the candidate node set $U$ is used to generate candidate path set Path under the condition of delay constraint. Step (9-17) is to select the shortest delay path in the Path as the optimal redeployment path $\boldsymbol{O R P}$. According to the $\boldsymbol{O R P}$, the resources of CPU, forwarding and link bandwidth are occupied to complete an SFC adjustment.

\section{Performance Evaluation}

In this paper, MATLAB is used for experimental simulation to verify the performance of the proposed method DA-SFC in a larger network, and the performance is compared with two other methods.

\subsection{Simulation environment settings}

This experiment takes the dynamic arrival, revocation and SFC dynamic adjustment in a relatively large network environment as the experimental background. The substrate network is composed of 100 nodes, whose connection probability is 0.5 , and SFC requests arrive dynamically. One SFC is selected and it contains 3 VNFs and the initial bandwidth demand is 10. The dynamic changes of network traffic within one experimental period of $T=150 \mathrm{~S}$ are predicted using the method proposed in literature [12], and the resource configuration is adjusted to meet the service demand according to the prediction. It is assumed that the change rate of network traffic passing through each VNF is $\alpha_{x}$, and the changes of network traffic will bring the same proportion change of VNF resource demand, forwarding resource demand and 
link resource demand. The service type of SFC is delay sensitive, and the number of VNFs is determined without changes. In order to reduce the impact of random factors, the experiment is conducted for 10 times, and the average value of the 10 experimental results is taken as the final experimental result.

\subsection{Experiment analysis}

In this paper, two groups of experiments are carried out to verify the performance of DASFC. In the first group of experiments, DA-SFC is used to compare with the other two methods under the same experimental environment, as shown in Table 1. The DM-SFC method in literature [24] is a coarse-grained adjustment method and the FGA-SFC method in [29] is a fine-grained adjustment method, both of them are the latest researches on dynamic adjustment. These two methods are selected as the comparison algorithms to verify the performance of DA-SFC. In the second group of experiments, the performance of DA-SFC is verified by changing the duration threshold $C^{-}$.

Table 1. Description of the three methods

\begin{tabular}{|c|l|}
\hline Method & \multicolumn{1}{c|}{ Description } \\
\hline DA-SFC & $\begin{array}{l}\text { First, the duration thresholds are set to generate the SFC dynamic adjustment requests } \\
\text { according to the network traffic prediction. Then, the SFC resource configuration is } \\
\text { adjusted by the hybrid approach of vertical scaling and migration, giving priority to } \\
\text { the vertical scaling. Finally, VNFs combination and VM reusing are fully considered } \\
\text { during the VNF migration. }\end{array}$ \\
\hline DM-SFC & $\begin{array}{l}\text { First, a network traffic prediction method is used to predict the resource adjustment } \\
\text { demand at the next moment. Then, a dynamic flow migration method is proposed to } \\
\text { dynamically migrate VNFs within the maximum allowable service interruption time, } \\
\text { so as to achieve a balance between network load balancing and adjustment operation } \\
\text { cost [24]. }\end{array}$ \\
\hline FGA-SFC & $\begin{array}{l}\text { First, a cloudscale method is used to predict the SFC resource configuration adjustment } \\
\text { demand. Then, the SFC resource configuration is adjusted by the hybrid approach of } \\
\text { vertical scaling and migration, giving priority to the vertical scaling [29]. }\end{array}$ \\
\hline
\end{tabular}

\subsubsection{Experiment 1: performance comparison}

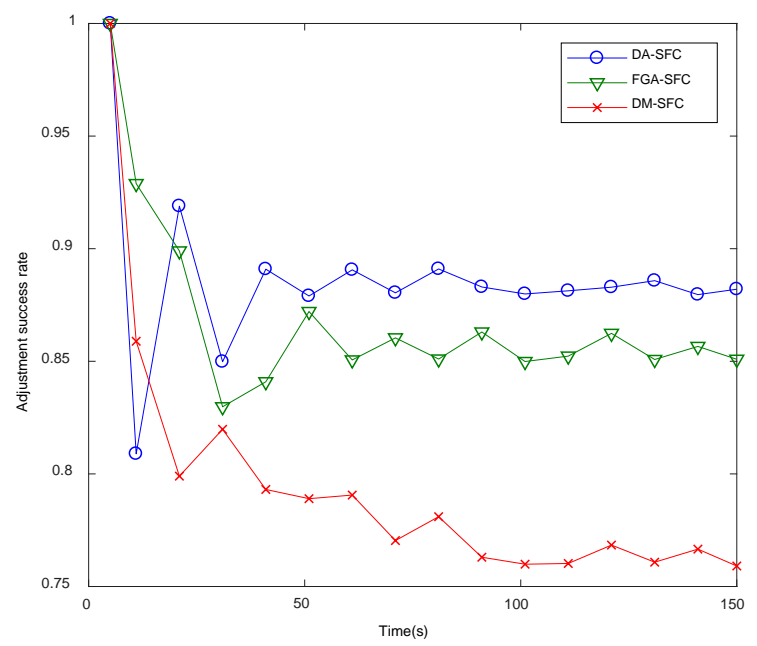

Fig. 4. Adjustment success rate 
As shown in Fig. 4, DM-SFC adopts VNF migration to process SFC dynamic adjustment requests, and the resource consumption is large, the migration frequency is high, and the adjustment success rate remains around 0.76 in a stable state. FGA-SFC uses a fine-grained approach to deal with the dynamic adjustment requests, but fails to consider the VNFs combination and VM reusing in the adjustment process, and the adjustment success rate remains around 0.85 in a stable state. The adjustment success rate of DA-SFC remains around 0.88 in a stable state, which is improved compared with the other two methods. This is due to the fine-grained method proposed in this paper and the full consideration of VNFs combination and VM reusing during the processing. The delay constraint is easier to meet and the adjustment success rate is improved.

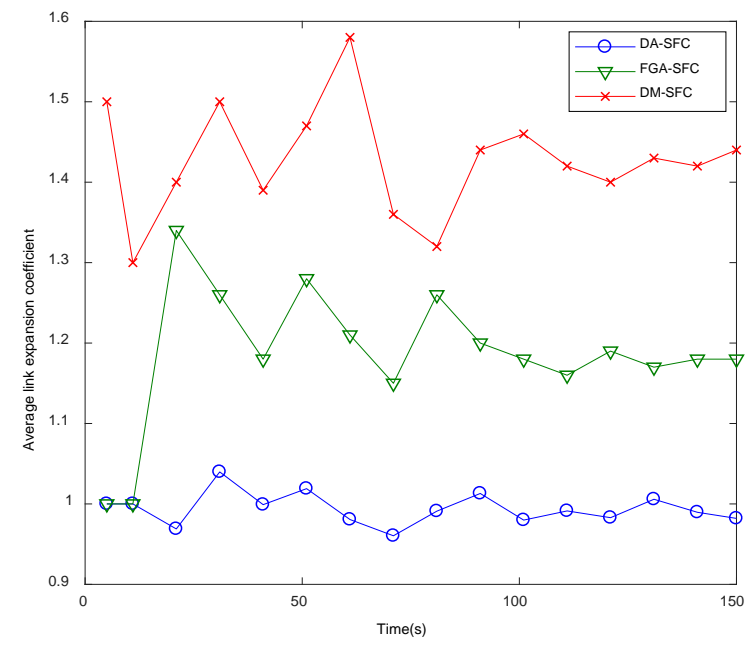

Fig. 5. Average link expansion coefficient

As shown in Fig. 5, DM-SFC adopts VNF migration to deal with the SFC dynamic adjustment requests, which will lead to longer deployment links, and the average link expansion coefficient remains around 1.44 in a stable state. FGA-SFC adopts the fine-grained method to deal with the dynamic adjustment request, and the average average link coefficient remains around 1.18 in the stable state. The average link expansion coefficient of DA-SFC remains around 0.98 in a stable state, and its performance is greatly improved compared with the other two methods. This is because DA-SFC is fine-grained for processing of dynamic SFC adjustment requests, and VNFs combination and VM reusing are fully considered in the process. Therefore, the resource utilization rate is greatly improved. 


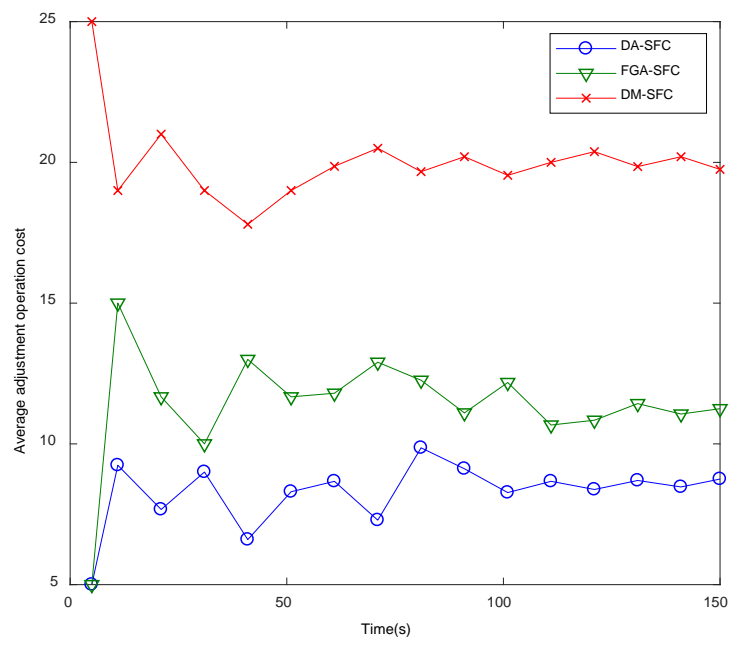

Fig. 6. Average adjustment operation cost

As shown in Fig. 6, DM-SFC adopts VNF migration to process SFC dynamic adjustment requests. The adjustment operation cost is relatively high, and the average adjustment operation cost remains around 19.75 in a stable state. FGA-SFC takes a fine-grained approach to deal with the dynamic adjustment requests. However, it fails to take into account the VNFs combination and VM reusing in the adjustment process, and the average adjustment operation cost remains around 11.25 in a stable state. Compared with DM-SFC, the average adjustment operation cost of FGA-SFC is lower. The average adjustment operation cost of DA-SFC remains at about 8.75 in a stable state, and its performance is greatly improved compared with the other two methods. This is because DA-SFC makes fine-grained processing of dynamic SFC adjustment requests and fully considers VNFs combination and VM reusing in the process. As a result, the adjustment operation cost is greatly reduced.

Table 2. Comparion of the average penalty

\begin{tabular}{|c|c|c|c|}
\hline Method & DA-SFC & DM-SFC & FGA-SFC \\
\hline Average penalty & 0.37 & 0.96 & 0.50 \\
\hline
\end{tabular}

As shown in Table 2, when the duration threshold $C^{-}$is $200 \mathrm{~ms}$, the average penalty of DASFC is 0.37 . Due to the network traffic prediction in advance and the proactive fine-grained adjustment of resource configuration, the service interruption time is negligible. As FGA-SFC predicts the resource demand in advance and takes the proactive strategy to make fine-grained adjustments to resource configuration, service interruption time is also negligible. However, due to the failure to consider VNFs combination and VM reusing in the migration process, the adjustment success rate is affected and the penalty of FGA-SFC is 0.50, which is higher than DA-SFC. As all the adjustment requests of DM-SFC are migrated online, the QoS is greatly affected and the penalty is increased compared with the above two methods. 


\subsubsection{Experiment 2 : duration threshold impact analysis on performance}

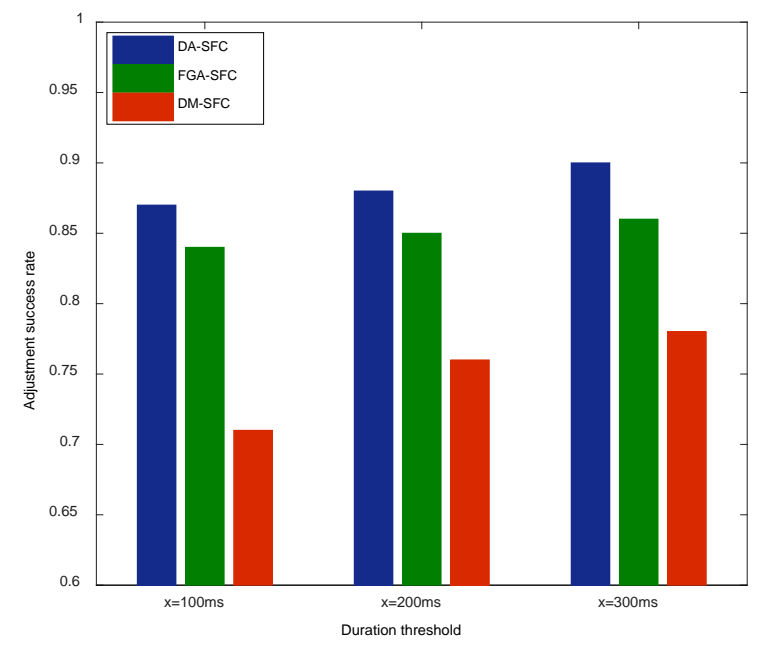

Fig. 7. Adjustment success rate

As shown in Fig. 7, when the duration threshold $C^{-}$are $100 \mathrm{~ms}, 200 \mathrm{~ms}$ and $300 \mathrm{~ms}$, respectively, the adjustment success rates of DM-SFC in the stable state are $0.71,0.76$ and 0.78 , respectively. The adjustment success rates of FGA-SFC are $0.84,0.85$ and 0.86 , respectively. The adjustment success rates of DA-SFC are $0.87,0.88$ and 0.90 , respectively. It can be seen that with the increase of the duration threshold $C$, the adjustment success rate of the three methods all increases to a certain extent. This is because the greater the duration threshold $C^{-}$is, the fewer adjustment requests are generated, and the adjustment success rate increases accordingly. Among the three methods, DA-SFC carries out fine-grained adjustment and fully considers VNFs combination and VM reusing, so the adjustment success rate remains high and the performance is the best.

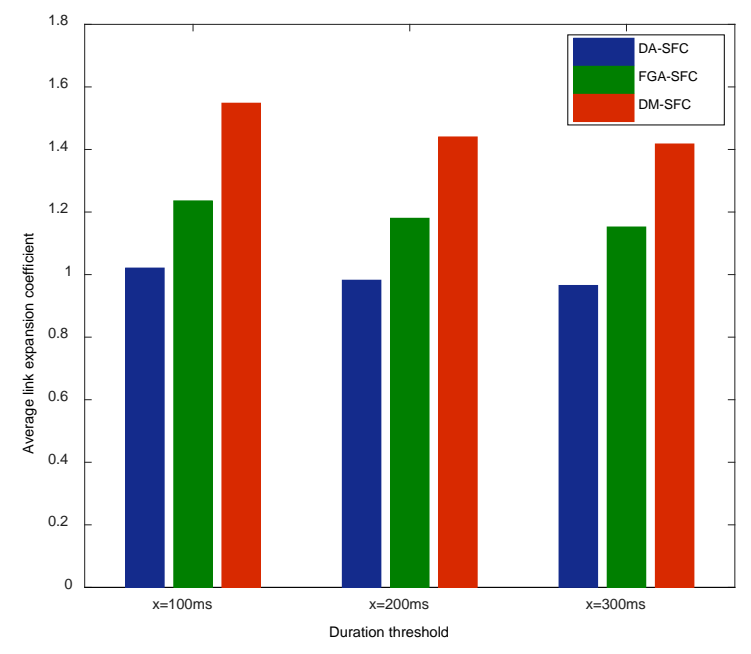

Fig. 8. Average link expansion coefficients 
As shown in Fig. 8, when the duration threshold $C^{-}$are $100 \mathrm{~ms}, 200 \mathrm{~ms}$ and $300 \mathrm{~ms}$, respectively, the average link expansion coefficients of DM-SFC in the stable state are 1.54, 1.44 and 1.41, respectively, and the average link expansion coefficients of FGA-SFC are 1.24, 1.18 and 1.15, respectively. The average link expansion coefficients of DA-SFC are 1.02, 0.98 and 0.97 , respectively. It can be seen that with the increase of the duration threshold $C^{-}$, the average link expansion coefficients of the three methods decrease accordingly. This is because the greater the duration threshold is, the fewer adjustment requests are generated. DA-SFC makes fine-grained adjustment and fully considers VNFs combination and VM reusing, so the average link expansion coefficient is low and the performance is the best in the three methods.

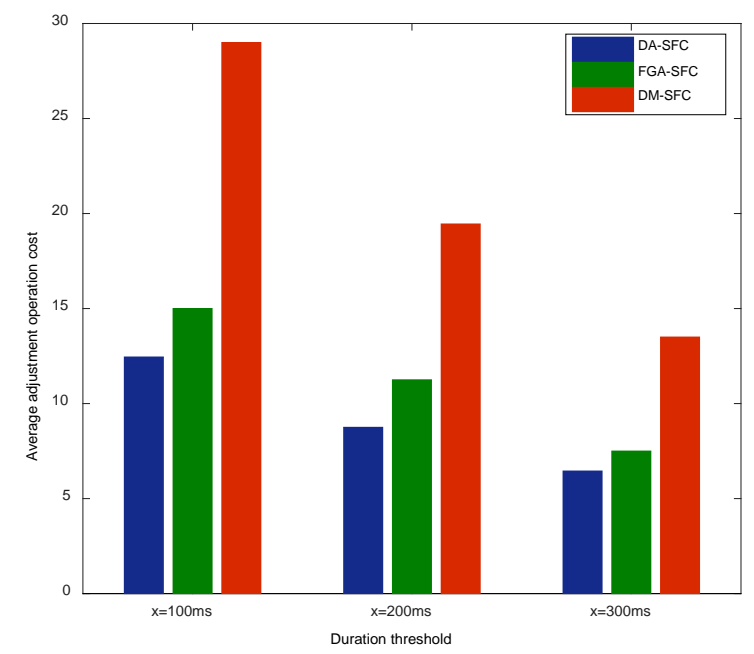

Fig. 9. Average adjustment operation cost

As shown in Fig. 9, when the duration thresholds $C^{-}$are $100 \mathrm{~ms}, 200 \mathrm{~ms}$ and $300 \mathrm{~ms}$, respectively, the average adjustment operation costs of DM-SFC in the stable state are 29.10, 19.45 and 13.50, respectively, and the average adjustment operation costs of FGA-SFC are 15.10, 11.25 and 7.5, respectively. The average adjustment operation costs of DA-SFC are $12.45,8.75$ and 6.45 , respectively. It can be seen that with the increase of the duration threshold $C^{-}$, the average adjustment operation costs of the three methods decrease accordingly. This is because the larger the duration threshold $C^{-}$is, the fewer adjustment requests are generated, and the average adjustment operation costs decrease. Among the three methods, DA-SFC carries out fine-grained adjustment and fully considers VNFs combination and VM reusing, so the average adjustment operation cost is always kept at the lowest, and its performance is the best in the three methods. 


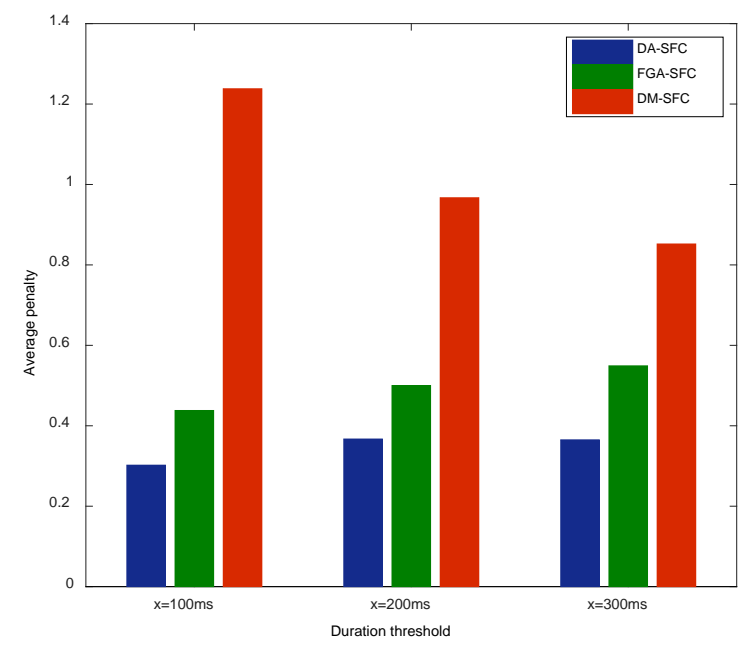

Fig. 10. Average penalty

As shown in Fig. 10, when the duration threshold $C^{-}$are $100 \mathrm{~ms}, 200 \mathrm{~ms}$ and $300 \mathrm{~ms}$, respectively, the average penalties of DM-SFC in the stable state are $0.365,0.549$ and 0.852 , respectively. The average penalties of FGA-SFC are $0.367,0.500$ and 0.967 , respectively. The average penalties of DA-SFC are 0.302, 0.438 and 1.238, respectively. When the duration threshold $C^{-}$gradually increases, it means that the QoS needs to be sacrificed to cope with the dynamic change of network traffic, and the penalty gradually increases. Experimental results show that with the increase of the duration threshold $C^{-}$, the penalties of FGA-SFC and DASFC keep increasing. On the contrary, DM-SFC will cause a large decline in QoS during the migration process. If the fluctuation duration is short and adjustment request is generated for adjustment, it will not only increase the adjustment operation cost, but also have a great impact on QoS. Therefore, the average penalty of DM-SFC can be reduced by increasing the duration threshold $C^{-}$.

From the above data analysis, it can be seen that DA-SFC always has a large advantage compared with the other two methods. The effects of duration threshold C- on average adjustment operation cost and average penalty are opposite. Therefore, choosing a reasonable duration threshold C- can keep a balance between average adjustment operation cost and average penalty and improve the revenue of service providers.

\section{Conclusion}

To cope with the dynamic adjustment of SFC resource configuration, this paper proposes an SFC dynamic adjustment method. First, the SFC dynamic adjustment request is generated according to the network traffic prediction. Then, an appropriate SFC adjustment strategy is selected according to the availability of the substrate network. Finally, the SFC resource configuration is adjusted according to the selected adjustment strategy, and VNFs combination and VM reusing are fully considered in this process to reduce the network operation cost and the substrate network resource consumption. Experimental results show that the DA-SFC proposed in this paper can reduce the average adjustment operation cost and improve the revenue of service providers, and has better performance compared with two other methods. In the next step, the deep learning will be used to improve the accuracy of network traffic prediction and further improve the performance of SFC dynamic adjustment. 


\section{Acknowledgement}

This work was supported by the National Natural Science Foundation of China (No.61873277), and by the Key R\&D Project of Shaanxi Province(2020GY-026).

\section{References}

[1] M. Otokura, K. Leibnitz, Y. Koizumi, D. Kominami, T. Shimokawa, and M. Murata, "Evolvable virtual network function placement method: mechanism and performance evaluation,” IEEE Transactions on Network and Service Management, 16(1), 27-40, 2019. Article (CrossRef Link)

[2] T. Han and N. Ansari, "A traffic load balance framework for software defined radio access networks powered by hybrid energy sources," IEEE/ACM Transactions on Network, 24(2), 10381051, 2016. Article (CrossRef Link)

[3] M. Wang, B. Cheng, and J. Chen, "Joint availability guarantee and resource optimization of virtual network function placement in data center networks," IEEE Transactions on Network and Service Management, 17(2), 821-834, 2020. Article (CrossRef Link)

[4] X. Han, X. Meng, Z. Yu, Q. Kang, and Y. Zhao, “A service function chain deployment method based on network flow theory for load balance in operator networks,” IEEE Access, 8(1), 9318793199, 2020. Article (CrossRef Link)

[5] M. Tajiki, S. Salsano, L. Chiaraviglio, M. Shojafar and B. Akbari, “Joint energy efficient and QoSaware path allocation and VNF placement for service function chaining," IEEE Transactions on Network and Service Management, 16(1), 374-388, 2019. Article (CrossRef Link)

[6] J. Wang, H. Qi, K. Li, and X. Zhou, "PRSFC-IoT: a performance and resource aware orchestration system of service function chaining for internet of things," IEEE Internet of Things Journal, 5(3), 1400-1410, 2018. Article (CrossRef Link)

[7] M. Ghaznavi, A. Khan, N. Shahriar, K. Alsubhi, R. Ahmed, and R. Boutaba, "Elastic virtual network function placement," in Proc. of 2015 IEEE 4th International Conference on Cloud Networking (CloudNet), Niagara Falls, Canada, pp. 255-260, Oct. 2015.

[8] S. Palkar, C. Lan, S. Han, K. Jang, A. Panda, S. Ratnasamy, L. Rizzo, and S. Shenker, "E2: a framework for nfv applications,” in Proc. of the 25th Symposium on Operating Systems Principles, pp. 121-136, Oct. 2015.

[9] H. Yu, J. Yang, C. Fung, R. Boutaba, and Y. Zhuang, "ENSC: multi-resource hybrid scaling for elastic network service chain in clouds," in Proc. of 2018 IEEE 24th International Conference on Parallel and Distributed Systems (ICPADS), Singapore, pp. 34-41, 2018.

[10] J. Liu, H. Shen and H. Hu, "Load-aware and congestion-free state management in network function virtualization,” in Proc. of 2017 International Conference on Computing, Networking and Communications (ICNC): Optical and Grid Computing, Santa Clara, USA, Jan. 2017.

[11] J. Zu, G. Hu, Y. Wu, D. Shao, and J. Yan, "Resource aware chaining and adaptive capacity scaling for service function chains in distributed cloud network," IEEE Access, 7(1), 157707-157723, 2019. Article (CrossRef Link)

[12] Y. Su, X. Meng, Z. Yu and Q. Kang, “Cognitive virtual network reconfiguration method based on traffic prediction and link importance,” IEEE Access, 7(1), 138915-138926, 2019. Article (CrossRef Link)

[13] “Kvm cpu hotplug,”. https://www.linux-kvm.org/page/CPUHotPlug.

[14] L. Popa, P. Yalagandula, S. Banerjee, and J. Mogul, "Elasticswitch: practical work-conserving bandwidth guarantees for cloud computing," in Proc. of the ACM SIGCOMM 2013 conference on SIGCOMM, pp. 351-362, Aug. 2013.

[15] “Open vswitch,”. https://openvswitch.org.

[16]B. Andrus, A. Autenrieth, S. Pachnicke, J. Olmos and I. Monroy, "Live migration downtime analysis of a VNF guest for a proposed optical FMC network architecture,” in Proc. of ITG Symposium on Photonic Networks, Leipzig, Germany, pp. 1-5, May. 2016.

[17] “Wind river," 2017. https://www.windriver.com/. 
[18] M. Mao and M. Humphrey, "A performance study on the vm startup time in the cloud," in Proc. of 2012 IEEE Fifth International Conference on Cloud Computing, Honolulu, USA, June 2012.

[19]F. Zhang, G. Liu., X. Fu and R. Yahyapour, "A survey on virtual machine migration: challenges, techniques, and open issues,” IEEE Communications Surveys \& Tutorials, 20(2), 1206-1243, 2018. Article (CrossRef Link)

[20] B. Yi, X. Wang, M. Huang and K. Li, "Design and implementation of network-aware VNF migration mechanism,” IEEE Access, 8(1), 44346-44358, 2020. Article (CrossRef Link)

[21] K. Noghani, A. Kassler, and J. Taheri, "On the cost-optimality trade-off for service function chain reconfiguration,” in Proc. of 2019 IEEE 8th International Conference on Cloud Networking (CloudNet), Coimbra, Portugal, Nov. 2019.

[22] S. Mehraghdam, M. Keller, and H. Karl, "Specifying and placing chains of virtual network functions," in Proc. of 2014 IEEE 3rd International Conference on Cloud Networking(CloudNet), Luxembourg, pp. 7-13, Oct. 2014.

[23] M. Pozza, K. Nicholson, D. Lugones, A. Rao, H. Flinck, and S. Tarkoma, "On reconfiguring 5G network slices," IEEE Journal on Selected Areas in Communications, 38(7), 1542-1554, 2020. Article (CrossRef Link)

[24] K. Qu, W. Zhuang, Q. Ye, X. Shen, X. Li, and J. Rao, "Dynamic flow migration for embedded services in SDN/NFV-enabled 5G core networks," IEEE Transactions on Communications, 68(4), 2394-2408, 2020. Article (CrossRef Link)

[25] L. Tang, X. He, P. Zhao, G. Zhao, Y. Zhou and Q. Chen, "Virtual network function migration based on dynamic resource requirements prediction,” IEEE Access, 7(1), 112346-112362, 2019. Article (CrossRef Link)

[26] H. Tang, D. Zhou, and D. Chen, "Dynamic network function instance scaling based on traffic forecasting and VNF placement in operator data centers," IEEE Transactions on Parallel and Distributed Systems, 30(3), 530-543, 2018. Article (CrossRef Link)

[27]Z. Luo, C. Wu, Z. Li, and W. Zhou, "Scaling geo-distributed network function chains: a prediction and learning framework," IEEE Journal on Selected Areas in Communications, 37(8), 1838-1850, 2019. Article (CrossRef Link)

[28] H. Yu, J. Yang, C. Fung, "Elastic network service chain with fine-grained vertical scaling," in Proc. of 2018 IEEE Global Communications Conference(GLOBECOM), Abu Dhabi, United Arab Emirates, Dec. 2018.

[29] H. Yu, J. Yang and C. Fung, "Fine-grained cloud resource provisioning for virtual network function," IEEE Transactions on Network and Service Management, 17(3), 1363-1376, 2020. Article (CrossRef Link) 

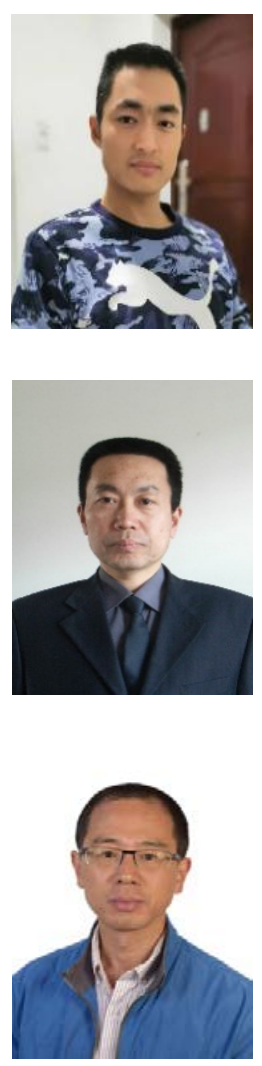

Zhenhua Yu received the B.S. degree and M.S. degree from Xidian University, Xi'an, China, in 1999 and 2003, respectively, and the Ph.D. degree from Xi'an Jiao Tong University, Xi'an, China, in 2006. He is currently a Professor with the Institute of Systems Security and Control, College of Computer Science and Technology, Xi'an University of Science and Technology, Xi'an, China. He has authored more than 20 technical papers for conferences and journals, and holds two invention patents. His research mainly focuses on cyber-physical systems, system security and social networks.

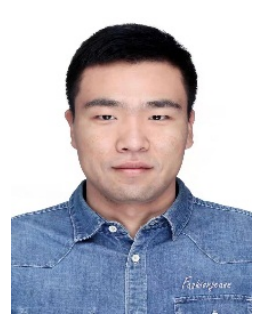

Xiaoyang Han was born in Zhumadian, Henan, China in 1986. He received his B.S. and M.S. degrees from Air Force Engineering University, China, in 2009 and respectively. Now, he is a Ph.D. candidate at Air Force Engineering University, China. His research interests include network function virtualization and network security.

Xiangru Meng was born in Lantian, Shannxi, China in 1963. He received his B.S., M.S. and Ph.D. degrees from Xi'an Jiaotong University, China in 1985, 1988 and 1994, respectively. He is currently a professor at Air Force Engineering University, Xi'an, China. From 1995 to 1997, he was a Visiting Scholar at the University of Electronic Science and Technology, Chengdu, China. His research interests include next generation Internet, network virtualization and survivable network.

Dong Zhai received the B.S. degree from Shanxi University, Taiyuan, China, in 2016, and the M.S. degree from Air Force Engineering University, Xi'an, China, in 2018. His research interests include network function virtualization and network security. 